\title{
Investigating drug-target interactions in frontotemporal dementia using a network pharmacology approach
}

\author{
Archana Balasubramanian, Raksha Sudarshan and Jhinuk Chatterjee * (1)
}

\begin{abstract}
Background: Frontotemporal dementia (FTD) is the second most common type of dementia in individuals aged below 65 years with no current cure. Current treatment plan is the administration of multiple medications. This has the issue of causing adverse effects due to unintentional drug-drug interactions. Therefore, there exists an urgent need to propose a novel targeted therapy that can maximize the benefits of FTD-specific drugs while minimizing its associated adverse side effects.

In this study, we implemented the concept of network pharmacology to understand the mechanism underlying FTD and highlight specific drug-gene and drug-drug interactions that can provide an interesting perspective in proposing a targeted therapy against FTD.
\end{abstract}

Results: We constructed protein-protein, drug-gene and drug-drug interaction networks to identify highly connected nodes and analysed their importance in associated enriched pathways. We also performed a historeceptomics analysis to determine tissue-specific drug interactions.

Through this study, we were able to shed light on the APP gene involved in FTD. The APP gene which was previously known to cause FTD cases in a small percentage is now being extensively studied owing to new reports claiming its participation in neurodegeneration. Our findings strengthen this hypothesis as the APP gene was found to have the highest node degree and betweenness centrality in our protein-protein interaction network and formed an essential hub node between disease susceptibility genes and neuroactive ligand-receptors.

Our findings also support the study of FTD being presented as a case of substance abuse. Our protein-protein interaction network highlights the target genes common to substance abuse (nicotine, morphine and cocaine addiction) and neuroactive ligand-receptor interaction pathways, therefore validating the cognitive impairment caused by substance abuse as a symptom of FTD.

Conclusions: Our study abandons the one-target one-drug approach and uses networks to define the disease mechanism underlying FTD. We were able to highlight important genes and pathways involved in FTD and analyse their relation with existing drugs that can provide an insight into effective medication management.

Keywords: FTD, Network pharmacology, Drug-gene network, Historeceptomics, Substance abuse, APP gene

${ }^{*}$ Correspondence: jhinukchatterjee@pes.edu

Department of Biotechnology, PES University, Bangalore, India

\section{Background}

Frontotemporal dementia (FTD) is a term given to a group of multi-factorial disorders responsible for youngonset dementia varied by their pathological, genetic and clinical heterogeneity [1]. These disorders result in pathogenic protein accumulation in tiny structures called Pick 
bodies resulting in selective destruction of brain networks, hence giving it the name Pick's disorder [2]. There is no holistic explanation for the variations in FTD but recent studies suggest that mutations in different genes result in behavioural changes, impaired judgment and lack of empathy which varies on a case-to-case basis [3].

Based on the clinical presentation and mutational status, FTD can be classified into 3 distinct types: behavioural variant FTD (bvFTD) [4], semantic variant primary progressive aphasia (svPPA) and nonfluent variant primary progressive aphasia (nfvPPA) [5].

In a FTD patient, the current treatment plan is the administration of multiple medications to manage the physically visible symptoms. This approach is not suitable to many patients and has various drawbacks primarily due to unintentional drug-drug interactions. In the past two decades, the pharmaceutical industry has developed and tested several drugs for psychiatric disorders, including FTD, but it still remains largely unclear the interactions between the drugs and their targeted genes and the possible adverse side effects caused by the interaction between the drugs themselves [6]. In this regard, our study aims to explore the mechanism of drug-gene and drug-drug interactions in FTD to suggest a novel targeted gene therapy. We used the principle of network pharmacology [7] to construct protein-protein, drug-gene and drug-drug interaction networks. We also identified FTD-specific hub nodes, deciphered enriched pathways, isolated potential gene biomarkers and elucidated possible off-target effects.

This study is important as it identifies specific cognitive, behavioural and genetic biomarkers responsible for FTD [8]. This can help future investigators in designing clinical trials and in the recommendation of precision medicine. Based on the type of FTD presented, a physician can suggest an effective treatment that takes into account genetic variability and individual neuropathology.

\section{Methods}

\subsection{Data retrieval}

The DrugBank database (version 5.1.8) [9] is a comprehensive drug database that provides information about drugs and their associated gene targets, indications and pathways. Other relevant information such as the chemical, pharmaceutical, mechanism of action, pharmacodynamics, toxicity, clinical trials and absorption, distribution, metabolism, excretion and toxicity (ADMET) properties of drugs are also listed.

To identify drugs related to FTD, "frontotemporal dementia" was used as a search term. For each drug, the information about its Food and Drug Administration (FDA) status, number of targets, target genes, Universal
Protein Resource (UniProt) ID of the target genes and DrugBank ID was retrieved.

NeuroDNet [10] an open-source platform that hosts a collection of disease models was used to collect the list of susceptible genes, their UniProt IDs and associated disorders linked to these genes.

\subsection{Functional enrichment analysis}

Functional enrichment analysis is a method used to identify pathways of statistical significance. This method recognizes the overrepresented genes and the sub-systems associated with it. This gives us a biological insight into the intersecting pathways that could be affected when a drug is administered. WebGestalt, a Web-based Gene Set Analysis Toolkit [11] was used to interpret and analyse the significantly enriched molecular pathways associated with the target genes.

To perform enrichment analysis, the following steps were followed:

-Homo sapiens, overrepresentation analysis (ORA), pathway and Kyoto Encyclopedia of Genes and Genomes (KEGG) were chosen as the organism of interest, method of enrichment analysis, functional database category and functional database name, respectively. Gene symbol was chosen as the gene ID type, and the list of target genes retrieved from DrugBank was imported into WebGestalt in Text (tab-delimited) format.

-Protein coding genome was selected as the reference gene set for enrichment analysis.

- The Benjamini-Hochberg (BH) procedure was used to control the false discovery rate (FDR) at 0.05 .

The enrichment results were displayed in the form of a bar chart (default) and a table in the decreasing order of enrichment ratio. The p-value and FDR values were set to 0.05 as a threshold for significance. The volcano plot is a $-\log 10 \mathrm{FDR} v / \mathrm{s}+\log 2$ enrichment value plot that can be downloaded to visualize the significantly enriched gene sets. Each gene set from the table was then downloaded in the form of a.csv file for further analysis.

\subsection{Protein-protein interaction using STRING}

To analyse the biological interaction between the target genes and the disease susceptibility genes, protein-protein interaction (PPI) network was constructed using the STRING (Search Tool for the Retrieval of Interacting Genes) v11 database. STRING is a biological database consisting of physical and functional protein-protein interactions derived from public text collections, experimental data, co-expression and genomic context predictions. A text file containing the gene targets (DrugBank) and the disease susceptibility genes (NeuroDNet) was imported into STRING [12]. 
The network was constructed based on the interaction data provided from text mining, experiments, databases, co-expression, neighbourhood, gene fusion and co-occurrence. The target genes of the drugs represent the nodes and the interactions between them represent the edges in the PPI network. STRING also provides the functional enrichments in a network that include a list of biological processes, molecular functions, cellular components, protein domains, etc. Each function is also provided with a count in the network, strength of interaction and the FDR. The Network Stats provided by STRING consists of the number of nodes, number of edges, average node degree, average local clustering coefficient, expected number of edges and the PPI enrichment $\mathrm{p}$-value. A very low $\mathrm{p}$-value indicates statistical significance. The biological significance of the network can be assessed by comparing the observed number of edges and the expected number of edges with a $p$ value of 0.05 as threshold. If the observed edge count is way higher than the expected edge count, it indicates that the proteins are biologically connected as a group and do not belong to a random set of proteins of similar size.

The interaction network obtained was updated by removing disconnected nodes and by increasing the confidence score to 0.700 (high confidence) to reduce the number of false positives. The resulting network was imported into Cytoscape [13] and was analysed based on the node degree and betweenness centrality values using the network analyser plug-in of Cytoscape.

A high node degree represents a greater interaction with other nodes, and a high betweenness centrality (based on shortest paths) indicates a better reach and connection within the network. These nodes can be classified as hub nodes that form bridges between clusters in a network. These hub nodes play an important role in network architecture and targeting them can result in the impairment of the entire network.

\subsection{Drug-gene network analysis and visualization using Cytoscape}

Cytoscape 3.8.2 is an open-source, publicly available tool written in Java that is used for network representation and analysis.

To visualize the drug-target interactions for the enriched categories, a Python script was written to read the.csv files downloaded for each enriched gene set from WebGestalt as mentioned earlier. The script mapped the UniProt IDs from each gene set with the respective target drugs from DrugBank as columns into individual.csv files. Each resultant.csv file was imported as networks into Cytoscape. The genes were chosen as source nodes and the drugs as target nodes. The edges correspond to the interaction between the nodes. The individual networks were merged and analysed based on the node degree and betweenness centrality. The "Style" option was used to change the shape of the drugs and gene targets to ellipse and round rectangle, respectively. The node colour was changed to match the node degree based on continuous mapping. The darker-coloured nodes represented a higher node degree while the lighter-coloured nodes represented a lower node degree.

\subsection{Historeceptomics approach}

To further evaluate the region of action of multi-target drugs and multi-drug combinations, a historeceptomics analysis was performed. Historeceptomics is a bioinformatics method that integrates tissue specificity with drug-target data.

One can narrow down the common tissues targeted by the FTD drugs and make an educated guess about possible drug-drug interaction at the tissue level using the historeceptomics profiler. Historeceptomics profiler [14] is a tool that assigns a historeceptomics (HR) score to each target-tissue pair of the input drug and ranks them based on the drug's likeness to elicit a phenotype in that tissue. The top five drugs with the highest node degrees were fed as input to the tool. The output table lists the possible targets (proteins/drug receptors) for the specific drug, corresponding tissues displaying the drug activity, target gene, its UniProt ID and the source of the drug (ChEMBL/DrugBank). The score section of the table consists of the Z-score (observed gene expression value in said tissue when compared with the mean of the gene expression values in other tissues), intensity (raw gene expression value), HR score (an amalgamation of drugtarget affinity and tissue expression values, a higher score indicates the higher likeness of the drug to bind to that tissue) and the $p$ value of the HR score. The common target tissues of the five drugs were tabulated and taken forward for further analysis.

\subsection{Drug-drug association network}

To analyse the adverse drug-drug interactions among the FTD drugs, drug interactions for each drug were collected from the Drug Interaction Lookup tool by DrugBank.

A Python script was written to extract only the interactions with the FTD drugs present in our drug list. The results were used to construct a drug-drug interaction network in Cytoscape. The drugs depicted as nodes while the edges depicted the adverse drug-drug interactions. The colours of the nodes were changed to reflect the node degree. The darker-coloured nodes represented more interactions in the network while the lighter-coloured nodes represented fewer interactions in the network. 


\section{Results}

From the DrugBank database, 55 FTD-related drugs along with their 87 gene targets were retrieved. Among these 55 drugs, 31 drugs are approved, 40 are still in the investigational stage, 2 are in the experimental stage and 3 are withdrawn. The details of the 55 FTD-related drugs, their DrugBank IDs, FDA approval status and the number of targets are given in Table 1. Based on the frequency of interaction, ACHE, DRD2, ADRA1A, HRH1 and BCHE were the top five target genes interacting with the largest number of FTD-related drugs.

From NeuroDNet, a list of 7 FTD susceptible genes were retrieved. Among these, the most prevalent are Microtubule Associated Protein Tau (MAPT) and Granulin Precursor (GRN) as they represent the autosomal dominant inheritance of FTD as given in Table 2.

Using WebGestalt, top 10 enriched pathways were generated. Based on the enrichment ratio, nicotine addiction (ratio $=55.419$ ) had the highest overlap between the observed and expected genes, followed by neuroactive ligand-receptor interaction $($ ratio $=18.889)$ and cocaine addiction $($ ratio $=18.096)$, whereas based on the expect value, neuroactive ligandreceptor interaction ranked 1 . Out of the 10 pathways, 3 pathways were related to substance abuse (nicotine, morphine and cocaine addictions) which suggests that substance abuse and prescription medications such as antinicotinic medication may act on pathways that contribute to cognitive impairment and result in symptoms of dementia and its sub-types [15]. A study also highlighted that exposure to multiple types of strong anticholinergic drugs is associated with an increased risk of dementia [16].

Neuroactive ligand-receptor interaction had the most number of genes (59) as it is involved in neuronal development. The $p$ values range from $(0.00$ to $1.0915 \mathrm{e}-10)$ and FDR values from ( 0.00 to $3.5582 \mathrm{e}-09)$. The FDR correction factor removes the false positives. The very low $\mathrm{p}$-values for the 10 biological pathways indicate that the genes grouped into them are not random but specific to the pathway. These pathways can be viewed in the KEGG database. The result of the enrichment analysis is given in Table 3.

The false discovery rate (FDR) and p-values were estimated by the Benjamini-Hochberg procedure. The enrichment ratio is calculated as the number of observed genes by the number of expected genes in each of the individual enriched gene sets obtained from KEGG.

To evaluate whether our genes in the PPI network were related, we compared the expected number of edges with the observed number of edges. The observed value (404) was significantly higher than the expected value (55) with the PPI enrichment $p$ value $<1.0 \mathrm{e}-16$, indicating that the target genes were interconnected and not by random chance.

Protein-protein interaction network showed that the disease susceptibility genes such as MAPT and PSEN1 interact with APP with a high confidence score of 0.949 and 0.998 , respectively. MAPT in turn interacts with the rest of the disease susceptibility genes (C9orf72, CHMP2B, GRN, PSEN1, TARDBP and VCP) making APP an essential link between disease susceptibility genes and drug target genes, especially neuroactive ligand-receptors. The target genes common to substance abuse (nicotine, morphine and cocaine addiction) and neuroactive ligand-receptor interaction pathways are the GABAergic genes and ionotropic glutamate receptor genes as shown in Fig. 1.

On analysing the network in Cytoscape, it was found that the APP gene had the highest node degree (31) and betweenness centrality (0.45) and formed an important PPI hub in the network. Other genes with high node degrees include HTR3A, DRD2, HTR1A, CHRM2 and GABRG2.

The individual drug-gene tables created for each of the 10 enriched pathways were imported and merged as networks in Cytoscape. From the Style settings, the gene target and drug node shapes were changed to round rectangle and ellipse, respectively, via discrete mapping. On analysing the merged network, few important statistics are as follows: number of nodes $=89$, number of edges $=149$ and average number of neighbours $=3.641$.

It was observed that the drugs memantine (node degree $=29, \mathrm{BC}=0.54$ ) and quetiapine (node degree $=26$, $\mathrm{BC}=0.39)$ had the highest node degree and betweenness centrality and hence, stood out in the merged network. Other drugs with high node degree include clonazepam, donepezil, risperidone, propiomazine and trazodone.

Two distinct clusters were identified with donepezil as the bridge drug between the two. Donepezil is an essential enzyme blocker used as a drug to restore the balance of neurotransmitters. It is one of the popular drugs, in combination with memantine, to treat dementia-related disorders. Clonazepam and memantine act as source drugs for GABA and GRN genes. GABA genes are inhibitory neurotransmitters while GRN genes are involved in neuronal proliferation and development. Heterozygous mutations in the GRN genes are a primary cause of FTD. Quetiapine, risperidone, propiomazine and trazodone form the source nodes for many target genes like the adrenergic, serotonergic and acetylcholine receptors. These drugs play a primary role in treating the neuropsychiatric symptoms in dementia and observing their interactions with target genes would be helpful in proposing a novel targeted gene therapy. The drug-target gene interaction network is displayed in Fig. 2. 
Table 1 Summary of frontotemporal dementia drugs, FDA status and number of targets based on DrugBank annotation

\begin{tabular}{|c|c|c|c|c|}
\hline SI. No & DrugBank ID & Generic name & FDA status & \# Targets \\
\hline 1 & DB00556 & Perflutren & Approved & - \\
\hline 2 & DB12401 & Bromperidol & Approved, investigational & No annotation \\
\hline 3 & DB01043 & Memantine & Approved, investigational & 7 \\
\hline 4 & DB00372 & Thiethylperazine & Approved, withdrawn & 1 \\
\hline 5 & DB00699 & Nicergoline & Approved, investigational & 1 \\
\hline 6 & DB01381 & Ginkgo biloba & Approved, investigational, nutraceutical & 6 \\
\hline 7 & DB01068 & Clonazepam & Approved, illicit & 3 \\
\hline 8 & DB00777 & Propiomazine & Approved & 6 \\
\hline 9 & DB00656 & Trazodone & Approved, investigational & 8 \\
\hline 10 & DB00620 & Triamcinolone & Approved, vet approved & 1 \\
\hline 11 & DB08842 & Acetylcarnitine & Approved, investigational & - \\
\hline 12 & DB00674 & Galantamine & Approved & 4 \\
\hline 13 & DB00382 & Tacrine & Approved, investigational, withdrawn & 3 \\
\hline 14 & DB06016 & Cariprazine & Approved, investigational & 6 \\
\hline 15 & DB12710 & Perazine & Approved, investigational & No annotation \\
\hline 16 & DB12867 & Benperidol & Approved, investigational & No annotation \\
\hline 17 & DB01121 & Phenacemide & Approved & 1 \\
\hline 18 & DB00377 & Palonosetron & Approved, investigational & 1 \\
\hline 19 & DB00122 & Choline & Approved, nutracitical & 8 \\
\hline 20 & DB00889 & Granisetron & Approved, investigational & 1 \\
\hline 21 & DB08870 & Brentuximab vedotin & Approved, investigational & 1 \\
\hline 22 & DB00904 & Ondansetron & Approved & 5 \\
\hline 23 & DB00433 & Prochlorperazine & Approved, vet approved & 4 \\
\hline 24 & DB00843 & Donepezil & Approved & 8 \\
\hline 25 & DB00989 & Rivastigmine & Approved, investigational & 2 \\
\hline 26 & DB01209 & Dezocine & Approved, investigational & 2 \\
\hline 27 & DB11584 & Pipradrol & Approved & 1 \\
\hline 28 & DB01224 & Quetiapine & Approved & 24 \\
\hline 29 & DB00734 & Risperidone & Approved, investigational & 13 \\
\hline 30 & DB09210 & Piracetam & Approved, investigational & - \\
\hline 31 & DB11239 & Aluminium sulphate & Approved & - \\
\hline 32 & DB13668 & Ipidacrine & Experimental & No annotation \\
\hline 33 & DB12969 & Methylinositol & Investigational & No annotation \\
\hline 34 & DB11756 & Solanezumab & Investigational & No annotation \\
\hline 35 & DB12034 & Gantenerumab & Investigational & No annotation \\
\hline 36 & DB04859 & Zanapezil & Investigational & 1 \\
\hline 37 & DB13084 & Pyritinol & Investigational & No annotation \\
\hline 38 & DB12958 & Prothipendyl & Investigational & No annotation \\
\hline 39 & DB06106 & AlT-034 & Investigational & No annotation \\
\hline 40 & DB12311 & CP1-1189 & Investigational & No annotation \\
\hline 41 & DB05432 & DAS-431 IV & Investigational & 1 \\
\hline 42 & DB12930 & Opipramol & Investigational & No annotation \\
\hline 43 & DB12555 & Nelotanserin & Investigational & 1 \\
\hline 44 & DB16214 & Landipirdine & Investigational & No annotation \\
\hline 45 & DB06504 & S-8510 & Investigational & No annotation \\
\hline 46 & DB11758 & Cenicriviroc & Investigational & No annotation \\
\hline 47 & DB05180 & LX6171 & Investigational & - \\
\hline 48 & DB06465 & FK-960 & Investigational & No annotation \\
\hline 49 & DB08831 & 2-deoxyglucose & Experimental, investigational & - \\
\hline
\end{tabular}


Table 1 (continued)

\begin{tabular}{lllll}
\hline SI. No & DrugBank ID & Generic name & FDA status & \# Targets \\
\hline 50 & DB06247 & CX516 & Investigational & 1 \\
51 & DB05814 & GPI-1485 & Investigational & 1 \\
52 & DB05450 & Smilagenin & Investigational & No annotation \\
53 & DB12613 & Davunetide & Investigational & No annotation \\
54 & DB04892 & Phenserine & Investigational & 3 \\
55 & DB12129 & Iideglusib & & 1 \\
\hline
\end{tabular}

Table 2 Summary of frontotemporal dementia susceptible genes and their associated disorders retrieved from NeuroDNet

\begin{tabular}{lll}
\hline FTD susceptible genes & Associated disorders & UniProt ID \\
\hline C9orf72 & Amyotrophic lateral sclerosis (OMIM: 105400) \\
& Frontotemporal dementia (OMIM: 600274) \\
CHMP2B & Frontotemporal dementia (OMIM: 600274) \\
GRN & Multiple sclerosis (OMIM: 126200) \\
& Frontotemporal dementia (OMIM: 600274) \\
MAPT & Supranuclear palsy (OMIM: 601104) \\
& Dementia, frontotemporal (OMIM: 600274) \\
& Dementia (OMIM: 600,274) \\
& Supranuclear palsy, progressive atypical (OMIM: 260,540), Pick disease (OMIM: 172,700), Parkinson \\
& disease (OMIM: 168,600), tauopathy and respiratory failure (OMIM: 157,140), amyotrophic lateral \\
& sclerosis (OMIM: 105,400) \\
& Frontotemporal dementia (OMIM: 600274) \\
& Frontotemporal dementia (OMIM: 600274) \\
& Disease (OMIM: 172700) \\
Amyotrophic lateral sclerosis (OMIM: 105400) & Alzheimer disease (OMIM: 104300) \\
PSEN1 & Cardiomyopathy dilated 1U (OMIM: 613694) \\
& Acne inversa (OMIM: 613737) \\
& Frontotemporal dementia (OMIM: 600274) \\
& Amyotrophic lateral sclerosis (OMIM: 105400) \\
& Frontotemporal dementia (OMIM: 600274) \\
& Frontotemporal dementia (OMIM: 600274) \\
& Frontotemporal dementia (OMIM: 600274) \\
Amyotrophic lateral sclerosis (OMIM: 105400) & P49768 \\
TARDBP &
\end{tabular}

Table 3 Enriched KEGG pathways in target genes of FTD drugs as identified by WebGestalt

\begin{tabular}{|c|c|c|c|c|c|c|}
\hline Gene Set & Description & Size & Expect & Ratio & $p$ value & FDR \\
\hline hsa04080 & Neuroactive ligand-receptor interaction & 277 & 2.9 & 19.9 & 0 & 0 \\
\hline hsa04024 & CAMP signalling pathway & 199 & 2.1 & 10.3 & 0 & 0 \\
\hline hsa04020 & Calcium signalling pathway & 183 & 1.9 & 11.2 & 0 & 0 \\
\hline hsa05032 & Morphine addiction & 91 & 0.9 & 18.4 & 0 & 0 \\
\hline hsa05033 & Nicotine addiction & 40 & 0.4 & 58.3 & 0 & 0 \\
\hline hsa04727 & GABAergic synapse & 88 & 0.9 & 16.9 & $4.4 \mathrm{E}-16$ & $2.4 \mathrm{E}-14$ \\
\hline hsa04726 & Serotonergic synapse & 115 & 1.2 & 12.9 & $4.0 \mathrm{E}-14$ & $1.9 \mathrm{E}-12$ \\
\hline hsa04723 & Retrograde endocannabinoid signalling & 148 & 1.6 & 10.7 & $1.5 \mathrm{E}-13$ & $6.2 \mathrm{E}-12$ \\
\hline hsa04742 & Taste transduction & 83 & 0.9 & 13.5 & $5.1 \mathrm{E}-11$ & $1.8 \mathrm{E}-09$ \\
\hline hsa05030 & Cocaine addiction & 49 & 0.5 & 19.0 & $6.5 \mathrm{E}-11$ & $2.1 \mathrm{E}-09$ \\
\hline
\end{tabular}




\section{(See figure on next page.)}

Fig. 1 Protein-protein interaction (PPI) network created using STRING. The nodes represent proteins and the edges represent protein-protein association. The edge confidence is set to high (0.700), and disconnected nodes are hidden in the network. The nodes common to the most enriched pathways, i.e., substance abuse receptors and neuroactive ligand-receptors, are highlighted. Nodes in blue represent nicotine addiction, nodes in green represent cocaine addiction, nodes in yellow represent morphine addiction and nodes in red represent neuroactive ligand-receptor interaction. The edges are coloured based on the following types of interactions: known interactions (turquoise — from curated databases, purpleexperimentally determined), predicted interactions (bright green—gene neighbourhood, red-gene fusions, dark blue-gene co-occurrence) and others (yellow - text mining, black — co-expression, light blue_protein homology). The filled nodes show that the 3D structure of the protein is known or predicted

The top 5 drugs with the highest node degree in the drug-target gene interaction network were subjected to historeceptomics analysis and the results were analysed as given in Table 4. It was found that memantine and clonazepam primarily target different regions of the brain such as the amygdala, cerebellum and occipital lobe, while quetiapine and donepezil target the heart and the skeletal muscles. Risperidone, on the other hand, targeted the thymus and the cells of the immune system.

Using the Drug Interaction Lookup tool from DrugBank, drug-drug interactions were visualized in Cytoscape. Based on the highest node degree, quetiapine (58), propiomazine (52), ondansetron (48), trazodone (46), risperidone (46) and donepezil (46) were the drugs with the highest interaction. This indicates that a combination of these drugs taken together could result in multiple adverse drug-drug interactions and severe side effects.

Another cause of concern is off-target effects. Offtarget effects refer to adverse effects as a result of drugs binding to an unrelated region of interest either due to resemblance to the predicted target or by modulating other targets. The top 6 hub drugs bind to various regions in the body: quetiapine, donepezil and propiomazine (skeletal and heart muscles), ondansetron and risperidone (thymus and liver) and trazodone (brain and liver) and when administered as a combination may result in off-target effects. Therefore, a thorough understanding of the mechanism of each drug and its interaction with other drugs is required to minimize adverse effects and to maximize the efficacy of the drugs. The drug-drug interaction network is displayed in Fig. 3.

\section{Discussion}

Out of the 55 FTD-related drugs retrieved from DrugBank, the drugs with the highest interaction with target genes were memantine, quetiapine, donepezil, risperidone and clonazepam. The expression of these target genes was significantly enriched in the brain, lymph, thymus, heart, liver and thyroid indicating the multi-target effect of these drugs. With the use of multitarget drugs comes the drawback of off-target effects. For effective drug management, it is crucial to select the right combination of target drugs and achieve the right balance between them while eliminating undesired target activity, especially in the case of targets belonging to the same family (kinase inhibitors) or having the same functional domain [17].

We performed pathway enrichment analysis to generate the top 10 enriched pathways of which 3 belonged to substance abuse. We also generated a PPI network that displayed the target genes common to substance abuse (nicotine, morphine and cocaine addiction) and neuroactive ligand-receptor interaction pathways to understand the relation between the pathways. The common target genes were the GABAergic and ionotropic glutamate receptor genes primarily responsible for cognition and behaviour. Our findings suggest that certain characteristics of the behavioural variant FTD (bvFTD) such as behavioural disinhibition and impulsivity could be manifested by substance misuse. Previous studies show alcohol and drug use as components preceding, following or accompanying bvFTD, thus substantiating our hypothesis. The past decade has seen a rise in substance abuse in the older population prone to cognitive disorders ranging from prescription misuse to illegal substance use. Clinical treatment providers are therefore being encouraged to monitor substance use patterns in all FTD sub-types [18].

Our study also highlights the importance of the APP gene in FTD which forms an essential link between disease susceptibility genes and drug target genes. Earlier studies restricted the role of the APP gene to inherited FTDs but are now being extensively studied with new reports claiming its participation in neurodegeneration and cognitive impairment. A reduction in APP-derived peptides is now known to affect the amyloidogenic pathway, neuropsychological performance, cortical thickness and cortical gene expression profiles in frontotemporal lobar degeneration [19]. Our findings strengthen this hypothesis as the APP gene was found to have the highest node degree (31) and betweenness centrality $(0.45)$ in our PPI network connecting various cholinergic, dopamine, opioid and adreno receptors that play an important role in neurodegeneration. The APP gene also behaves as a biomarker in identifying specific cognitive and 


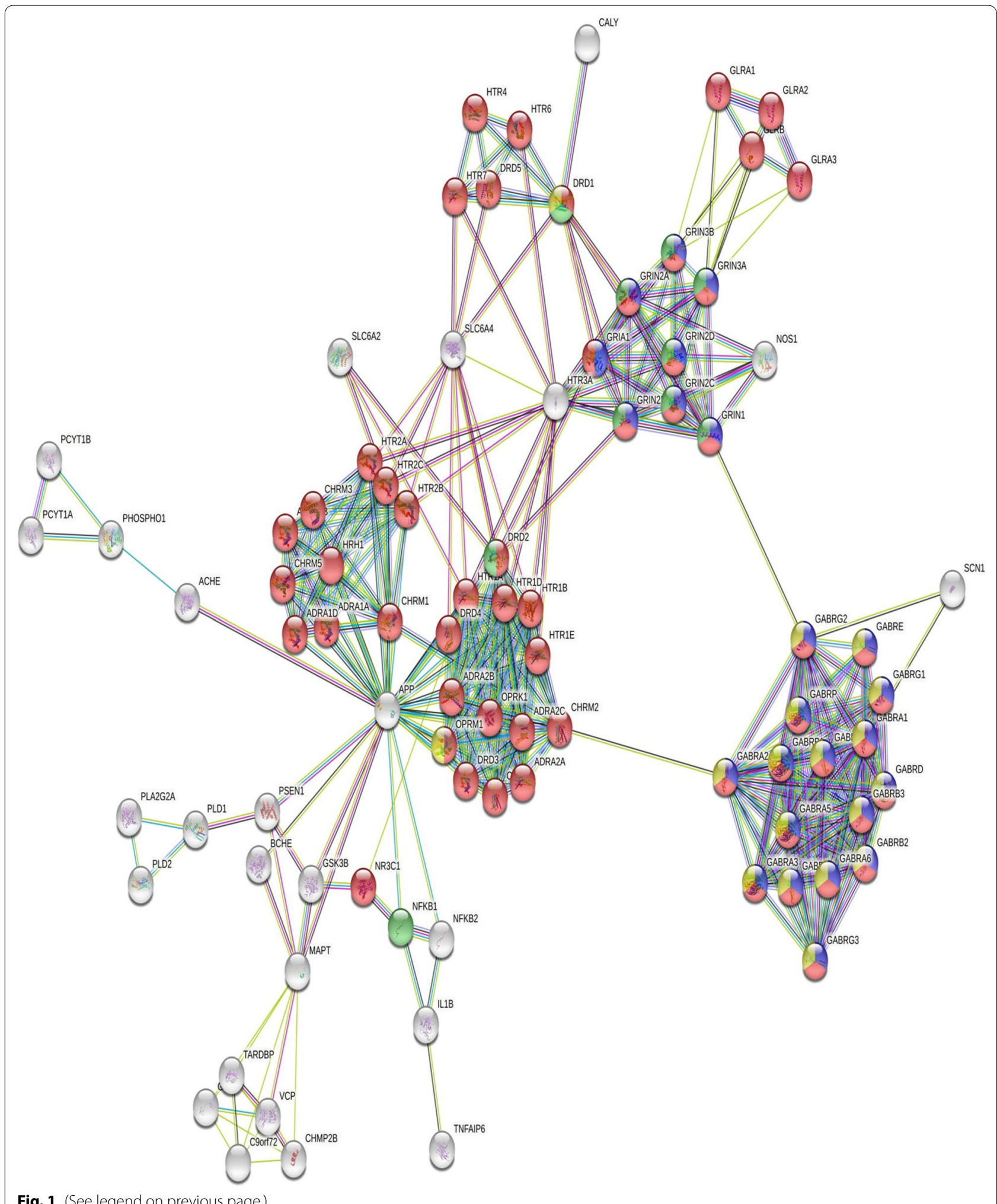

Fig. 1 (See legend on previous page.) 


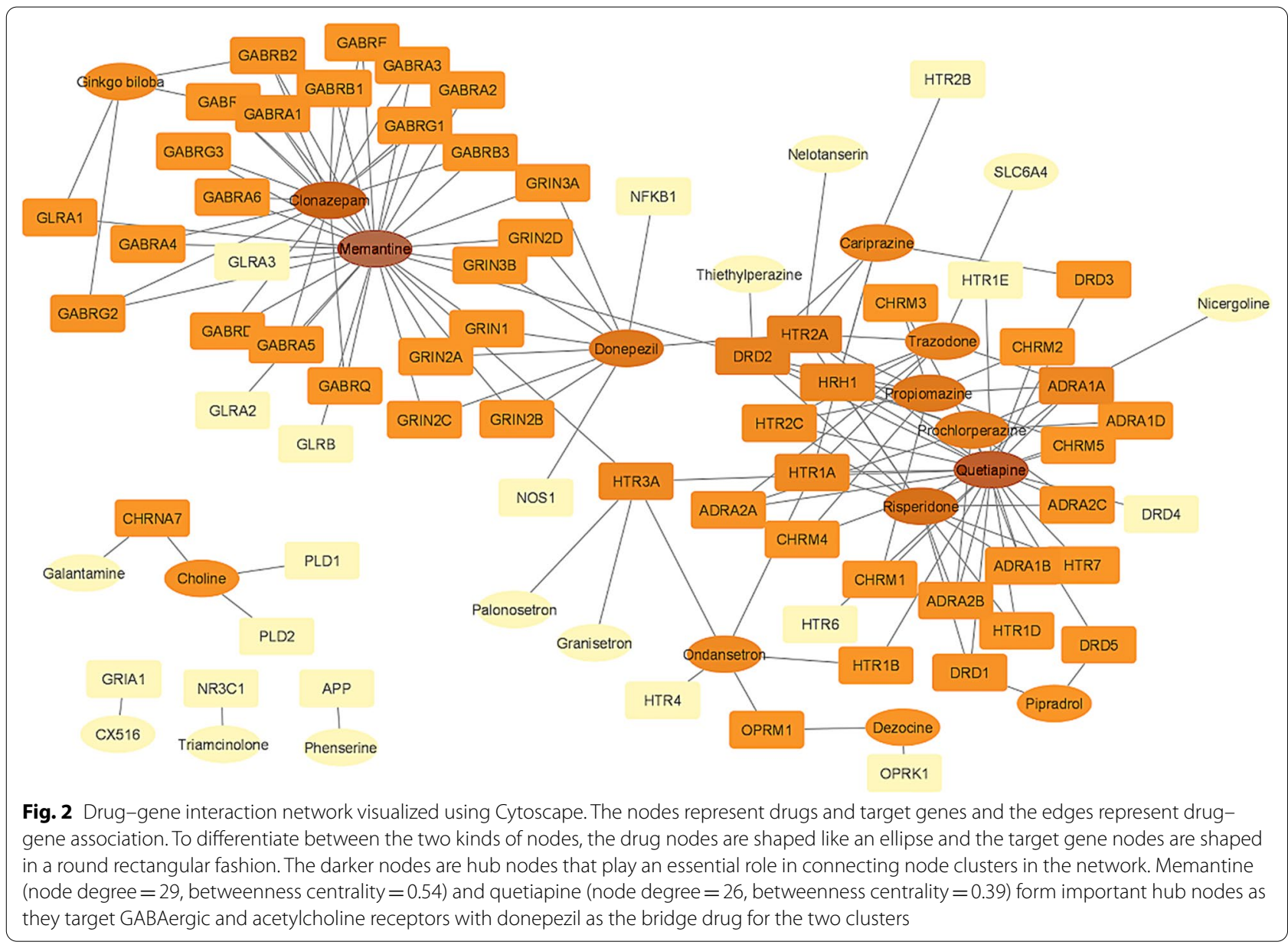

behavioural aspects of FTD. In this regard; it would be interesting to observe the APP-phenserine interaction in the drug-gene network. Phenserine has a low node degree in the drug-drug interaction network, making it an ideal candidate to effectively manage the symptoms of FTD while minimizing its associated adverse side effects.

Effective medication administration is key in the symptomatic management of FTD due to its varying clinical presentations and its overlap with other neurodegenerative syndromes such as amyotrophic lateral sclerosis (ALS), Parkinson's and Alzheimer's. Previous therapies include N-methyl-D-aspartic acid (NMDA) receptor antagonists, cholinesterase inhibitors, selective serotonin reuptake inhibitors, antipsychotics, dopamine modulating therapeutics, antiepileptic agents, etc. failed at various stages. Each therapy had its drawbacks either due to major drug interactions or adverse side effects with little to no symptomatic improvement. The relationship between FTD and neurodegenerative disorders such as ALS and Parkinson's has gained recognition over the past few years with over 15\% of FTD patients displaying signs of ALS. Recent advances in understanding the underlying mechanism behind FTD have led to the development of novel targeted therapies at the gene level. Mutations in MAPT (tau) and GRN (progranulin) genes are responsible for FTD-associated Parkinsonism while mutations in the C9orf72 gene are responsible for FTD-ALS. As emphasized earlier, our findings display the APP gene as an important hub linking disease susceptible genes (C9orf72, CHMP2B, GRN, MAPT, PSEN1, TARDBP and $\mathrm{VCP}$ ) with neuroactive ligand receptors making it an important gene to be targeted in developing a potentially disease-modifying FTD therapy [20].

A major limitation in clinical trials is the lack of information on genetic variability and drug-gene interactions. In the current world of polypharmacy, developing physiologically based pharmacokinetic (PBPK) and drugdrug-gene interaction (DDGI) models can help in the real-time prediction of drug interactions and their possible outcomes [21]. Our study can assist future investigators in incorporating these factors in building reliable models to test clinical studies.

With the advancement in precision medicine, attempts on targeted therapeutics are made based on 


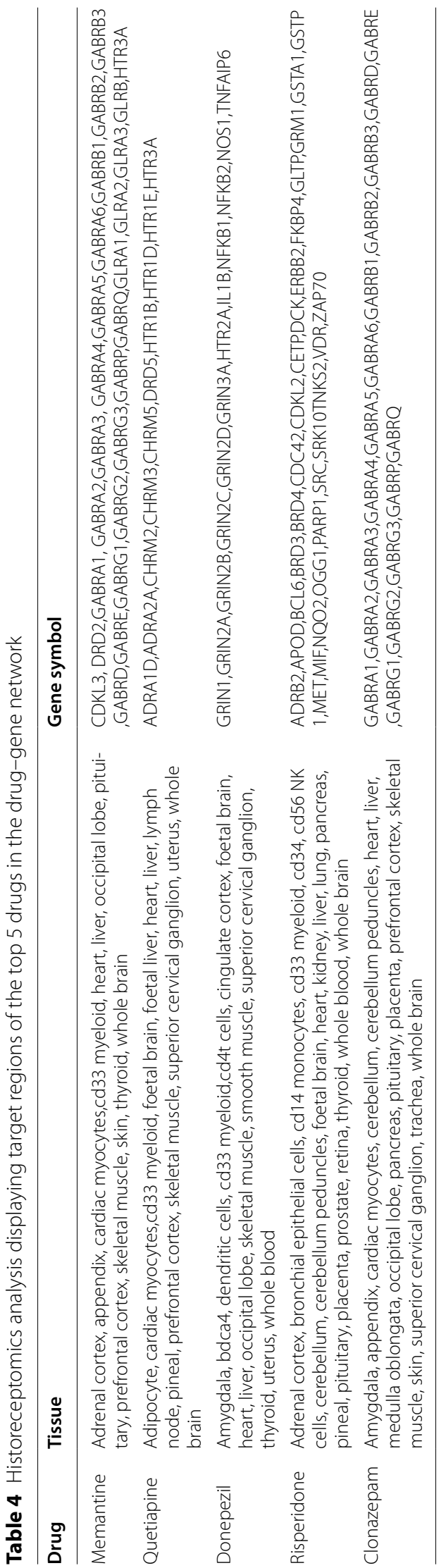




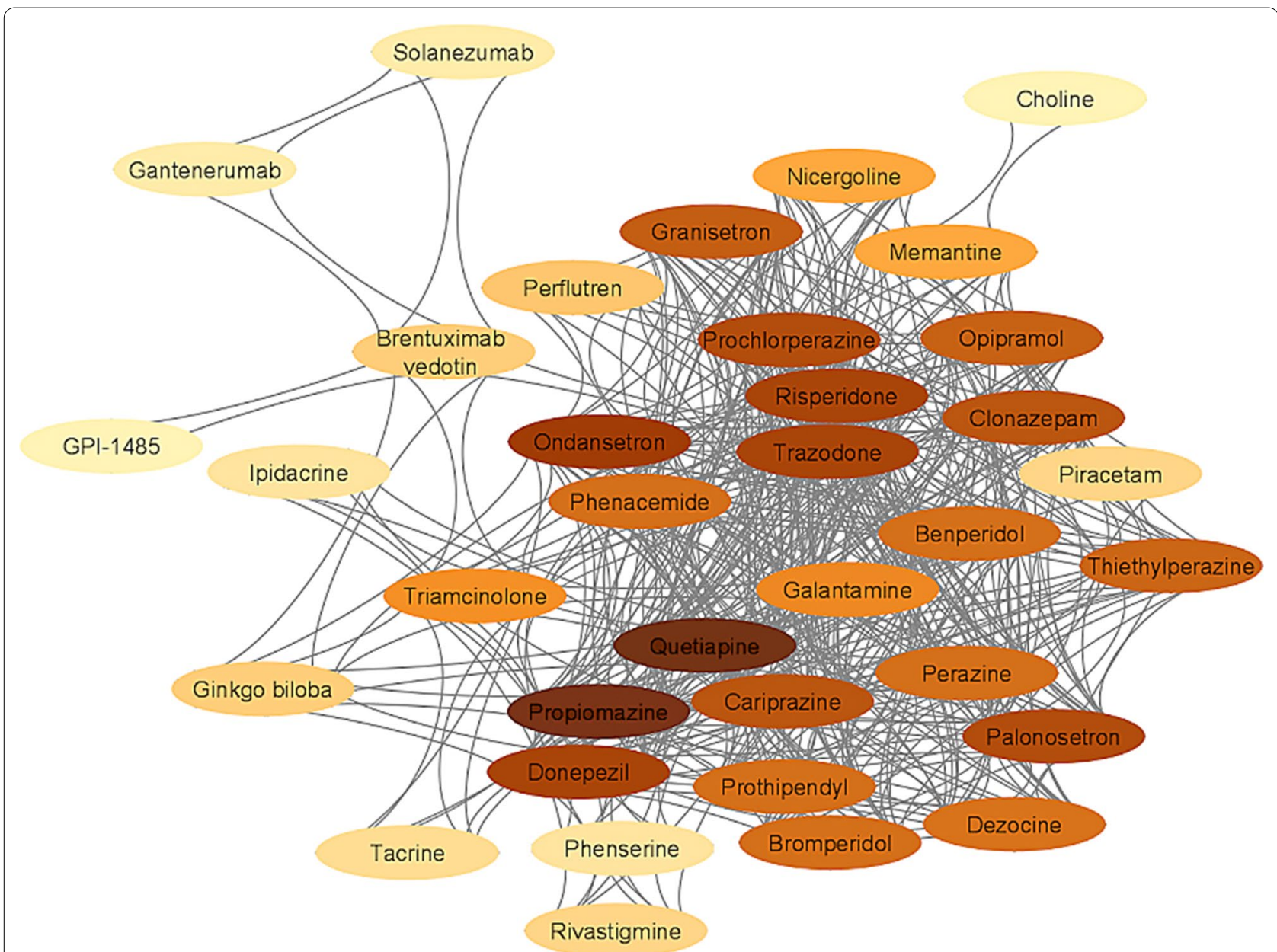

Fig. 3 Drug-drug interaction network visualized using Cytoscape. The nodes represent drugs and the edges represent drug-drug association. The darker nodes are hub nodes with high node degree. These drugs may eventually result in adverse side effects when administered as a combination. Quetiapine (node degree $=58$ ) and propiomazine (node degree $=52$ ) are the drugs that had the highest interaction in this FTD drug interaction network

an individual's genetic risk variant and type of FTD [22]. These include tau-targeting therapy for bvFTD patients, progranulin-related therapies for GRN mutation, antisense therapeutics to reduce C9orf72 repeat expansions, etc. On the clinical forefront, a future perspective of this study could involve the use of neuroimaging alongside specific biomarkers in classifying homogenous groups for testing targeted therapies [23].

With the increase in drug databases, future work can also involve the creation of a custom database that is regularly updated on FDA-approved drugs. Additionally, study can be expanded from FTD-related drugs to other related disorders such as ALS, Parkinson's and Alzheimer's to observe the similarity in the drug-gene network and identify sub-phenotypes.

\section{Conclusions}

Recent advances in the understanding of FTD have shown genetic factors to play an important role in its onset and progression. A complete framework of druggene and drug-drug interactions can help in the identification of potential biomarkers for innovative clinical trials. In this regard, our study gives an insight into the underlying mechanism of frontotemporal dementiarelated drugs, their mode of action on target genes and possible drug-drug interactions.

We identified 55 FTD-related drugs, 87 gene targets and 7 FTD susceptible genes from databases such as DrugBank and NeuroDNet. An enrichment and PPI network analysis revealed the gene targets overrepresented in neuroactive ligand-receptors and substance abuse systems (nicotine, 
cocaine and morphine) demonstrating that neuropsychiatric drugs do not function independently but rather forms an interacting network. We also identified the APP gene as an important hub node that forms an essential link between disease susceptibility genes and drug target genes.

In addition, we proved the off-target effects commonly seen in neuropsychiatric drugs by displaying their multitarget behaviour via a historeceptomics analysis. Our study shows that the network-based method is useful and might help in the development of an effective drug management approach from a computational biology perspective.

\begin{abstract}
Abbreviations
FTD: Frontotemporal dementia; APP: Amyloid precursor protein; bvFTD: Behavioural variant FTD; sVPPA: Semantic variant primary progressive aphasia; nfvPPA: Nonfluent variant primary progressive aphasia; ADMET: Absorption, distribution, metabolism, excretion and toxicity; FDA: Food and Drug Administration; UniProt: Universal Protein Resource; WebGestalt: Web-based Gene Set Analysis Toolkit; ORA: Overrepresentation analysis; KEGG: Kyoto Encyclopedia of Genes and Genomes; BH: Benjamini-Hochberg; FDR: False discovery rate; PPI: Protein-protein interaction; STRING: Search Tool for the Retrieval of Interacting Genes; HR: Historeceptomics; MAPT: Microtubule-Associated Protein Tau; GRN: Granulin Precursor; OMIM: Online mendelian inheritance in man; ALS: Amyotrophic lateral sclerosis; NMDA: N-Methyl-D-aspartic acid.
\end{abstract}

\section{Acknowledgements}

\section{Not applicable}

\section{Authors' contributions}

$A B$ had contributed to conceptualization, methodology, investigation and writing draft manuscript; RS was involved in conceptualization, methodology, investigation and writing draft manuscript. JC took part in conceptualization, supervision and manuscript finalization. All authors have read and approved the final manuscript.

\section{Funding}

Not applicable.

\section{Declarations}

Ethics approval and consent to participate:

Not applicable.

\section{Consent for publication}

Not applicable.

\section{Availability of data and materials}

The datasets generated and/or analysed during the current study are available in the [http://www.drugbank.ca/, https://bioschool.iitd.ac.in/NeuroDNet/, http://www.webgestalt.org/, https://historeceptomics.com, https://go.drugb ank.com/interax/drug_lookup]. ${ }^{\left[\left[g^{-}\right.\right.} 11 \cdot 14^{]]}$

\section{Competing interests:}

The authors declare that they have no competing interests.

Received: 9 June 2021 Accepted: 5 September 2021

Published online: 16 September 2021

\section{References}

1. Waldö ML (2015) The frontotemporal dementias. Psychiatr Clin North Am 38(2):193-209
2. Sivasathiaseelan $H$, Marshall CR, Agustus JL, Benhamou E, Bond RL, van Leeuwen JEP, Hardy CJD, Rohrer JD, Warren JD (2019) Frontotemporal Dementia: A Clinical Review. Semin Neurol 39(2):251-263

3. Maxime M, Brambati SM, Luisa G-T, Raffaella M (2018) Clinical, Anatomical, and Pathological Features in the Three Variants of Primary Progressive Aphasia: A Review. Front Neurol 9:692. https://doi.org/10.3389/ fneur.2018.00692

4. Lanata SC, Miller BL (2016) The behavioural variant frontotemporal dementia (bvFTD) syndrome in psychiatry. J Neurol Neurosurg Psychiatry 87(5):501-511

5. Bang J, Spina S, Miller BL (2015) Frontotemporal dementia. Lancet 386(10004):1672-1682

6. Panza F, Lozupone M, Seripa D, Daniele A, Watling M, Giannelli G, Imbimbo BP (2020) Development of disease-modifying drugs for frontotemporal dementia spectrum disorders. Nat Rev Neurol 16(4):213-228

7. Chandran U, Mehendale N, Patil S, Chaguturu R, Patwardhan B(2017) Network Pharmacology. Innovative Approaches in Drug Discovery:127-164. doi:https://doi.org/10.1016/B978-0-12-801814-9.00005-2.

8. Tsai RM, Boxer AL (2016) Therapy and clinical trials in frontotemporal dementia: past, present, and future. J Neurochem 138:211-221

9. Wishart DS, Feunang YD, Guo AC, Lo EJ, Marcu A, Grant JR, Sajed T, Johnson D, Li C, Sayeeda Z, Assempour N, lynkkaran I, Liu Y, Maciejewski A, Gale N, Wilson A, Chin L, Cummings R, Le D, Pon A, Knox C, Wilson M(2017) DrugBank 5.0: a major update to the DrugBank database for 2018. Nucleic Acids Res 46(D1): D1074-D1082.

10. Vasaikar, S.V., Padhi, A.K., Jayaram, B. James Gomes (2013) NeuroDNet - an open source platform for constructing and analyzing neurodegenerative disease networks. BMC Neurosci, 14, Article Number 3.

11. Liao Y, Wang J, Jaehnig EJ, Shi Z, Zhang B (2019) WebGestalt 2019: gene set analysis toolkit with revamped UIs and APIs. Nucleic Acids Res 47(W1):W199-W205

12. Szklarczyk D, Gable AL, Lyon D, Junge A, Wyder S, HuertaCepas J, Simonovic M, Doncheva NT, Morris JH, Bork P, Jensen LJ, Mering C (2019) STRING v11: protein-protein association networks with increased coverage, supporting functional discovery in genome-wide experimental datasets. Nucleic Acids Res 47(D1):D607-D613

13. Shannon P, Markiel A, Ozier O, Baliga NS, Wang JT, Ramage D, Amin N, Schwikowski B, Ideker T (2003) Cytoscape: a software environment for integrated models of biomolecular interaction networks. Genome Res 13(11):2498-2504

14. Shmelkov E, Grigoryan A, Swetnam J, Xin J, Tivon D, Shmelkov S, Cardozo T (2015) Historeceptomic fingerprints for drug-like compounds. Front Physiol 6:371. https://doi.org/10.3389/fphys.2015.00371

15. Reddy PV, Anuroop L, Shetageri V, Kumar RK, Gopalakrishnan G (2019) A Case of Frontotemporal Dementia Presenting as Nicotine Dependence and Carbohydrate Craving. Indian J Psychol Med 41(4):391-393

16. Coupland CAC, Hill T, Dening T, Morriss R, Moore M, Hippisley-Cox J (2019) Anticholinergic Drug Exposure and the Risk of Dementia: A Nested Case-Control Study. JAMA Intern Med 179(8):1084-1093

17. Decker M (2017) Design of hybrid molecules for drug development. Elsevier, Amsterdam

18. Kalapatapu RK, Delucchi KL, Wang S, Harbison JD, Nelson EE, Kramer $J H$ (2016) Substance use history in behavioral-variant frontotemporal dementia versus primary progressive aphasia. J Addict Dis 35(1):36-41. https://doi.org/10.1080/10550887.2015.1102026

19. Illán-Gala I, Pegueroles J, Montal V, Alcolea D, Vilaplana E, Bejanin A, Borrego-Écija S, Sampedro F, Subirana A, Sánchez-Saudinós MB, Rojas-García R, Vanderstichele H, Blesa R, Clarimón J, Antonell A, Lladó A, Sánchez-Valle R, Fortea J, Lleó A (2019) APP-derived peptides reflect neurodegeneration in frontotemporal dementia. Ann Clin Transl Neurol 6(12):2518-2530. https://doi.org/10.1002/acn3.50948

20. Boxer AL, Mackenzie IR, Boeve BF, Baker M, Seeley WW, Crook R, Feldman H, Hsiung GY, Rutherford N, Laluz V, Whitwell J, Foti D, McDade E, Molano J, Karydas A, Wojtas A, Goldman J, Mirsky J, Sengdy P, Dearmond S, Miller BL, Rademakers R (2011) Clinical, neuroimaging and neuropathological features of a new chromosome 9p-linked FTD-ALS family. J Neurol Neurosurg Psychiatry 82(2):196-203

21. Malki MA, Pearson ER (2020) Drug-drug-gene interactions and adverse drug reactions. Pharmacogenomics J 20(3):355-366 
22. Liu MN, Lau Cl, Lin CP (2019) Precision Medicine for Frontotemporal Dementia. Front Psychiatry 10:1-13

23. Borroni B, Benussi A (2019) Recent advances in understanding frontotemporal degeneration. F1000Res 8:F1000

\section{Publisher's Note}

Springer Nature remains neutral with regard to jurisdictional claims in published maps and institutional affiliations.
Submit your manuscript to a SpringerOpen ${ }^{\odot}$ journal and benefit from:

- Convenient online submission

- Rigorous peer review

- Open access: articles freely available online

- High visibility within the field

- Retaining the copyright to your article

Submit your next manuscript at $\boldsymbol{\nabla}$ springeropen.com 